\title{
Infrared studies of protein-induced perturbation of lipids in lipoproteins and membranes
}

\author{
Jose Luis R. Arrondo *, Félix M. Goñi \\ Departamento de Bioquímica, Grupo Biomembranas (Unidad Asociada al CSIC), Universidad del País Vasco, Aptdo. 644, \\ 48080 Bilbao, Spain
}

Received 4 March 1998; accepted 4 March 1998

\begin{abstract}
The paper reviews the main recent publications concerning infrared (IR) spectroscopy as applied to the study of lipid-protein interactions in model and cell membranes, lipoproteins, and related systems (e.g. lung surfactant). The review focuses mainly on transmission IR. Based on the available data, a number of general conclusions are presented on the perturbations caused by proteins on either the hydrocarbon chains, the polar headgroups or the interface region. Lipid-protein interactions in native cell membranes do not reveal significant differences from what is observed in semisynthetic model systems. (C) 1998 Elsevier Science Ireland Ltd. All rights reserved.
\end{abstract}

Keywords: Protein-induced perturbation; Lipoproteins; Infrared spectroscopy; Lipid-protein interactions; Biomembranes

\section{Introduction}

The term 'lipids' encompasses a wide variety of molecules whose capacity to self-aggregate in a multiplicity of architectures (polymorphism) is only matched by the plurality of their roles in biology, their 'poly-functionalism'. In their different missions, lipids often associate not only with themselves, but with other classes of biomolecules, giving rise to supramolecular associations of

\footnotetext{
* Corresponding author.
}

many sorts. Chief among these are the lipoproteins and the biological membranes. In all these structures the lipids are held together noncovalently by hydrophobic forces, although the specific geometries are often the result of a subtle equilibrium between hydrophobic and polar interactions. The link between lipids and proteins may be of different kinds: mainly covalent, as in the case of proteins with lipid anchors (that will remain beyond the scope of this review), mainly hydrophobic, as in intrinsic membrane proteins, or mainly polar, as in extrinsic or peripheral membrane proteins. 
The interactions between lipids and proteins are as complex as the molecules involved. The early studies were mainly concerned with the effect of intrinsic proteins on the surrounding phospholipids (Chapman et al., 1979), although it was soon realized that lipids influence protein conformation as much as proteins influence lipids (Chapman et al., 1982). The multiplicity of systems explored in the subsequent years, including non-integral membrane proteins, proteins that interact only transiently with membranes, and the various lipoproteins, have contributed to enrich our view of the picture as well as, perhaps, to blur some its contours (Watts and DuPont, 1986; Watts, 1993). The present situation is one in which each system under study appears to hide its own peculiarity, and the optimistic generalisations of the seventies have been substituted by the sober acceptance of an amazing variability, hardly kept together by a few of the 'traditional' general physical principles (Tanford, 1980).

In the present state of knowledge infrared spectroscopy appears as a useful tool in the study of lipid-protein interactions. The technological advances, both in the instruments (detectors, sources) and in the software (instrument control, data handling) have been considerable in the last

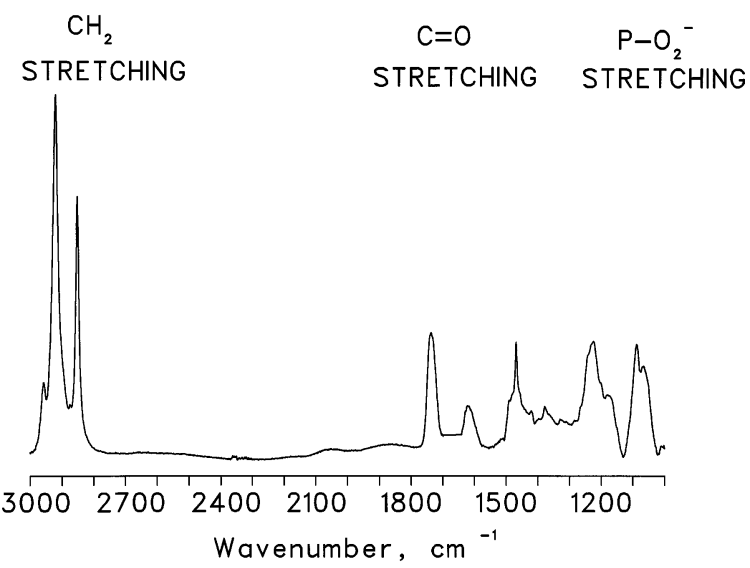

Fig. 1. Infrared spectrum of an aqueous suspension of a typical membrane lipid, dipalmitoylphosphatidylcholine, in the range $3000-1000 \mathrm{~cm}^{-1}$. The three regions of study corresponding to the hydrophobic region $\left(\mathrm{CH}_{2}\right.$ stretching), the interfacial region $(\mathrm{C}=\mathrm{O}$ stretching) and the polar region $(\mathrm{P}-$ $\mathrm{O}_{2}^{-}$stretching) are depicted in the Figure.
Table 1

Assignments of the lipid bands in the infrared spectrum of a membrane preparation ${ }^{\mathrm{a}}$

\begin{tabular}{|c|c|}
\hline $\begin{array}{l}\text { Wavenumber } \\
\left(\mathrm{cm}^{-1}\right)\end{array}$ & Assignment \\
\hline 2957 & $\mathrm{CH}_{3}$ stretching, asymmetric \\
\hline 2924 & $\mathrm{CH}_{2}$ stretching, asymmetric \\
\hline 2871 & $\mathrm{CH}_{3}$ stretching, symmetric \\
\hline 2853 & $\mathrm{CH}_{2}$ stretching, symmetric \\
\hline 1732 & $\mathrm{C}=\mathrm{O}$ stretching, esters \\
\hline 1467 & $\begin{array}{l}\mathrm{CH}_{2} \text { bending, scissoring }\left(\mathrm{L}_{\mathrm{c}}+\mathrm{L}_{\beta} \text { gel }\right. \\
\text { phase })\end{array}$ \\
\hline 1456 & $\begin{array}{l}\mathrm{CH}_{2} \text { bending, scissoring }\left(\mathrm{L}_{\mathrm{c}}+\mathrm{L}_{\beta} \text { gel }\right. \\
\text { phase })\end{array}$ \\
\hline 1402 & $s n-1, \alpha-\mathrm{CH}_{2}$ bending, scissoring \\
\hline 1380 & $\mathrm{CH}_{3}$ bending, deformation, symmetric \\
\hline 1233 & $\mathrm{PO}_{2}^{-}$stretching, asymmetric \\
\hline 1171 & $\mathrm{C}-\mathrm{O}$ stretching, single bond \\
\hline 1159 & $\mathrm{C}-\mathrm{C}$ stretching, skeletal \\
\hline 1060 & $\mathrm{R}-\mathrm{O}-\mathrm{P}-\mathrm{O}-\mathrm{R}^{\prime}$ \\
\hline 1082 & $\mathrm{PO}_{2}^{-}$stretching, symmetric \\
\hline
\end{tabular}

${ }^{\text {a }}$ Data corresponding to the spectrum of Fig. 1.

decade. The evolution of new or improved IR conformations (IR microscopy, reflectance techniques, continuous flow) leads to a constant increase in the number of objects and processes that become amenable to study by this technique. This review will deal with the applications of IR techniques to study the effect of proteins on the lipid structure and dynamics in biological membranes and lipoproteins. Taking into account the accompanying papers in this Special Issue, our review will focus primarily on transmission IR. Practical considerations make us also discuss with more detail those contributions appeared after our previous review on this topic (Arrondo and Goñi, 1993).

\section{Phospholipid band assignment}

The IR spectra of a typical phospholipid, phosphatidylcholine, dispersed in excess water is shown in Fig. 1. Phospholipid infrared bands in the spectrum can be divided into three different regions corresponding to the hydrophobic, interfacial and polar moieties of the lipid (Fringeli and Gunthard., 1981; Casal and Mantsch, 1984; Lee 
and Chapman, 1986; Mantsch and McElhaney, 1991). The characteristic vibrations of covalently bonded atoms can be classified as 'stretching', which involves changes in bond lengths, or 'bending', corresponding to changes in bond angles. Table 1 shows the assignments of the phospholipid peaks in the spectrum of Fig. 1.

\subsection{Acyl chain vibrational modes}

Bands arising from hydrophobic acyl residues have been easily assigned by comparing phospholipid bands to those of fatty acyl esters and other polymethylene chain compounds (Bellamy, 1980; Mendelsohn and Mantsch, 1986). C-H stretching vibrations give rise to bands in the 3100-2800 $\mathrm{cm}^{-1}$ region. Asymmetric and symmetric $\mathrm{CH}_{2}$ bands, at 2920 and $2850 \mathrm{~cm}^{-1}$, respectively, are the strongest ones in a phospholipid spectrum. These bands are also most useful in the study of the physical properties of phospholipids. Vibrational bands corresponding to terminal methyl residues, $\mathrm{CH}_{3}$, are found around $2956 \mathrm{~cm}^{-1}$ (asymmetric stretching) and $2870 \mathrm{~cm}^{-1}$ (symmetric stretching) appearing as shoulders of the stronger methylene bands. Olefinic group bands, $=\mathrm{C}-\mathrm{H}$, arising from unsaturated hydrophobic chains are usually located around $3010 \mathrm{~cm}^{-1}$.

Methylene bending bands (scissoring) are located around $1470 \mathrm{~cm}^{-1}$ and can split into two components in motion restricted chains. The $\mathrm{CH}_{2}$ wagging progression is found between 1380 and $1180 \mathrm{~cm}^{-1}$ in the gel phase of saturated phospholipids as a series of shoulders on the phosphate band (Mendelsohn and Mantsch, 1986). The methyl symmetric deformation mode around 1378 $\mathrm{cm}^{-1}$, that was assumed to be insensitive to changes in lipid morphology, was later found as sensitive to cochleate phase formation in phosphatidylserine-containing model membranes (Flach and Mendelsohn, 1993). Full or partial isotopic substitution of the acyl chains can be used to avoid interference with other components, i.e. in lipid mixtures or with proteins in lipidprotein interaction studies. In addition, selective deuteration can be used as an internal probe to study the characteristics of a specific methylene (Sunder et al., 1981). Asymmetric and symmetric
$\mathrm{CD}_{2}$ vibrational bands are located around 2195 and $2090 \mathrm{~cm}^{-1}$ respectively, whereas $\mathrm{CD}_{3}$ bands are found at 2212, 2169 and $2070 \mathrm{~cm}^{-1}$ (Castresana et al., 1991).

\subsection{The interfacial region}

The most prominent bands arising from the interfacial region are due to stretching vibrations of the carbonyl group involved in ester bonds, in the region $1750-1700 \mathrm{~cm}^{-1}$. These bands are responsive to changes in their environment, i.e. hydrogen bonding or polarity. Moreover, these vibrations occur in a region free of significant absorption by other infrared-active groups, mainly the amide I vibrational band of peptides and proteins, so they are of great interest. In dipalmitoylphosphatidylcholine (DPPC) this region consists of a broad band contour that appears to be the summation of two underlying components easily resolvable by deconvolution or derivation, giving maxima near 1743 and 1728 $\mathrm{cm}^{-1}$. In principle, these two bands were assigned to the $\mathrm{C}=\mathrm{O}$ stretching vibrations of the $s n-1$ and $s n-2$ positions of the glycerolipid moiety, respectively (Levin et al., 1982). The assignment was based on the study of dry or poorly hydrated DPPC. However, with the use of fully hydrated, specifically ${ }^{13} \mathrm{C}=\mathrm{O}$ labelled phospholipid bilayers, this assignment was questioned, since, should the two bands correspond to the $s n-1$ and $s n-2$ carbonyls, their maxima should not be more than 4 $\mathrm{cm}^{-1}$ apart. In fact, ${ }^{13} \mathrm{C}$ labelling of only one the positions still gave rise to two bands around 1743 and $1728 \mathrm{~cm}^{-1}$ (Blume et al., 1988; Lewis and McElhaney, 1992). These studies concluded that the underlying components normally resolved in the stretching vibrations of $\mathrm{C}=\mathrm{O}$ absorption bands are the summation of comparable contributions from both of the ester carbonyl groups and assigned to subpopulations of free and hydrogenbonded ester carbonyl groups. More recently, a re-evaluation of all the existing data has concluded that the values obtained for the dry or poorly hydrated samples cannot be translated to fully hydrated phospholipid and that instead of different bands arising from the $s n-1$ and $s n-2$ bonds, the bands are due to distinct hydrated 
subpopulations of both carbonyls (Lewis et al., 1994).

\subsection{Headgroup bands}

The most characteristic vibrational bands of the headgroup are those arising from the phosphate. Particularly, three bands appear in the $1300-1000 \mathrm{~cm}^{-1}$ region of a hydrated DPPC bilayer. As in the interfacial region, these bands are strongly dependent on the hydration state of the lipid and are sensitive to hydrogen bonding. The antisymmetric $\mathrm{PO}_{2}^{-}$stretching mode appears around $1240 \mathrm{~cm}^{-1}$ in 'dry' phosphate and shifts to around $1220 \mathrm{~cm}^{-1}$ in fully hydrated DPPC. The symmetric $\mathrm{PO}_{2}^{-}$stretching mode appears around $1086 \mathrm{~cm}^{-1}$ in hydrated DPPC. A shoulder near $1060 \mathrm{~cm}^{-1}$ has been attributed to a $\mathrm{R}-\mathrm{O}-\mathrm{P}-\mathrm{O}-\mathrm{R}^{\prime}$ stretching mode, which is equivalent to a $\mathrm{P}-\mathrm{O}-\mathrm{C}$ vibration with nonequivalent substituents (Arrondo et al., 1984). In choline and ethanolamine glycerophospholipids there are characteristic infrared bands due to these group vibrations, but they are of no significant diagnostic value (Casal and Mantsch, 1984).

\section{Membrane lipid perturbation by intrinsic proteins}

Perturbations introduced by proteins in membrane phospholipids may be studied by looking at the bands arising either from the acyl chain region, the interfacial region, or the polar head group. However, most studies on the effect of polypeptides and proteins on the physical state of the membrane lipids have been carried out using the $\mathrm{C}-\mathrm{H}$ (or $\mathrm{C}-\mathrm{D}$ ) asymmetric or symmetric stretching bands.

Glycophorin from human erythrocyte membranes, a well-characterized transmembrane protein, was first studied in relation to the changes induced in DPPC acyl chains. At different lipid/protein ratios, a picture in which the protein induces a broadened gel-to-liquid-crystal phase transition, while the pretransition is abolished, can be obtained, also showing a progressive increase in bandwidth in both the gel and liquid-crystal phases as more protein is added, which can be interpreted in terms of increased rates of acyl chain motion in the presence of protein (Mendelsohn et al., 1981). Similar results were obtained by Chapman and co-workers (Lee and Chapman, 1986), for the proteins $\mathrm{Ca}^{2+}-\mathrm{AT}$ Pase and bacteriorhodopsin, reconstituted in vesicles of DMPC or DPPC (Fig. 2). Below $T_{\mathrm{m}}$, these molecules cause an increase in the proportion of gauche isomers, whereas above $T_{\mathrm{m}}$, and mainly at high lipid/protein ratios, a reduction in the proportion of gauche conformers with respect to the pure lipid bilayer is observed (Cortijo et al., 1982).

Qualitatively similar effects are seen when phospholipids other than PC are used; e.g. glycophorin nearly abolishes the transition in PS vesicles, showing more pronounced effects than in DPPC, perhaps due to electrostatic interactions with positively charged regions of the protein (Surewicz et al., 1987a). The influence of the phospholipid headgroup has also been shown for the interaction of the polypeptide hormone calcitonin with two acidic phospholipids, DMPG and DMPA; the overall effect is again similar, but the influence of calcitonin on the conformation of acyl chains in DMPA is much smaller than in DMPG (Surewicz et al., 1987b).

The influence of the degree of unsaturation of phospholipid acyl chains has also been taken into account. A survey of the interaction of $\mathrm{Ca}^{2+}$-ATPase with different lipid classes (Anderle and Mendelsohn, 1986) has shown that (i) phospholipids with high levels of acyl chain unsaturation (DOPC or native SR lipids) have their acyl chains slightly ordered by $\mathrm{Ca}^{2+}$-ATPase; (ii) phospholipids with saturated acyl chains show slightly lowered melting temperatures and reduced cooperativity of melting upon $\mathrm{Ca}^{2+}$-ATPase interaction; and (iii) in phospholipids with one saturated and one unsaturated chain, or heterogeneous systems with low levels of unsaturation (egg sphingomyelin), a stronger effect of the protein on the acyl chains is seen, indicating the importance of unsaturation levels.

The studies by Gonzalez-Ros and co-workers (Castresana et al., 1992; Fernandez-Ballester et al., 1994), on the influence of cholesterol on the structure and stability of the nicotinic acetyl- 


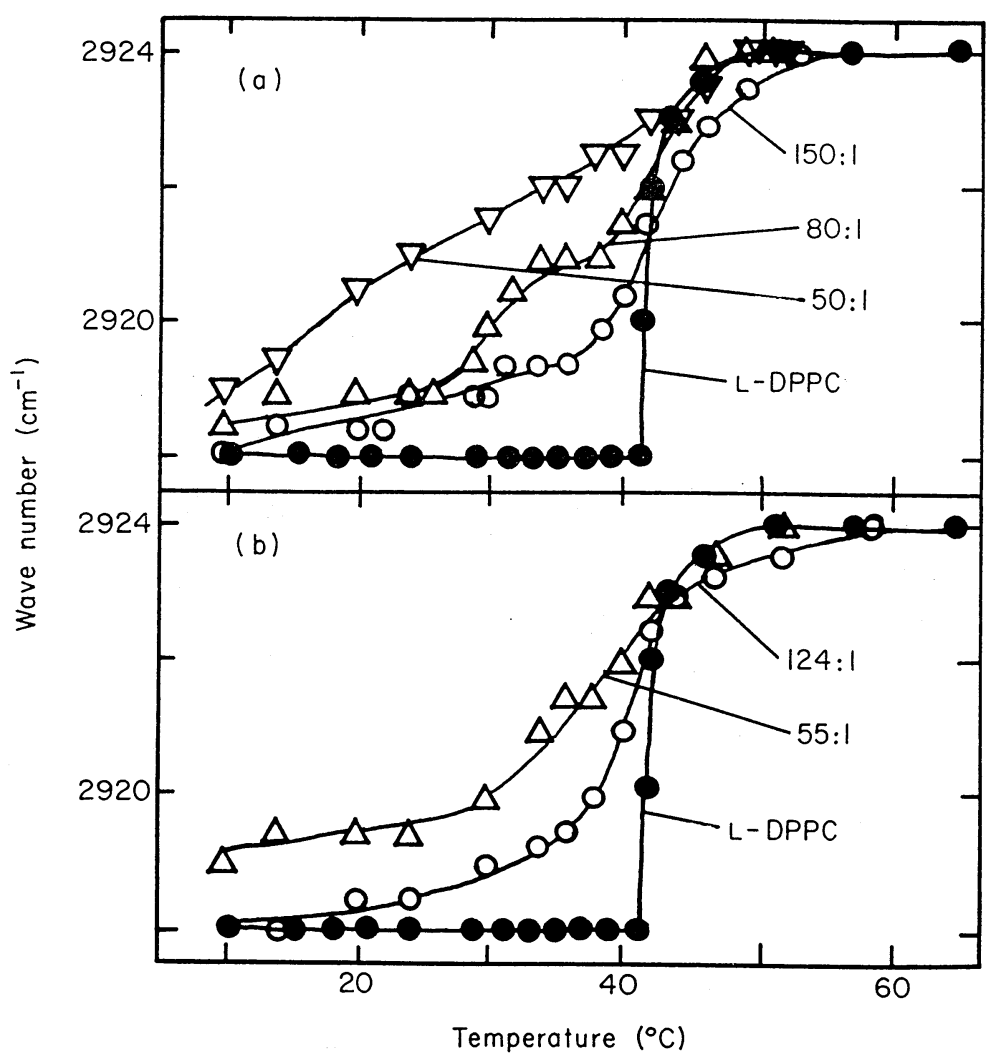

Fig. 2. Temperature dependence of the maximum wavenumber of the $\mathrm{CH}_{2}$ asymmetric stretching vibrations in (a) L-DPPC: $\mathrm{Ca}^{2+}$-ATPase; and (b) L-DPPC: bacteriorhodopsin at the molar ratios indicated. The temperature dependence for the pure lipids is also shown (Cortijo et al., 1982).

choline receptor deserve a mention here although the main subject of this review is rather the opposite, i.e. the effect of proteins on lipid structure. Their work is important because it stands probably alone in the IR literature demonstrating the interaction of a sterol with an intrinsic membrane protein.

\section{Membrane lipid perturbation by peripheral membrane proteins and soluble proteins}

Peripheral or extrinsic membrane proteins are very similar to those that exist largely unrelated to membranes, at least from the experimental point of view, since both classes can be purified and stored in the absence of detergents. There is probably a continuum from the extrinsic proteins that are invariably found associated to membranes, through the soluble ones that may still interact with lipids under certain conditions, to those that can never be induced to bind membranes. The early IR studies on lipid-protein interaction, reflecting the then predominant trends in membrane research, were mainly devoted to the effect of intrinsic proteins, as described in the previous section. More recent data, however, deal mainly with less tightly bound proteins.

An example of extrinsic membrane protein is the so-called myelin basic protein, which accounts for about 10\% (by weight) of myelin isolated from brain white matter. In addition to its high content of basic amino acids, it also has a certain degree of apolar character, in common with other extrinsic proteins e.g. apocytochrome $c$, that accounts for its interaction with the lipids. The myelin basic 
protein perturbs the lipid bilayers in various ways, and a variety of observations suggests that its interaction with the membrane is not limited to the polar part of the bilayer, but that instead it is also interacting with the lipid acyl chains (Surewicz et al., 1987a; Boggs et al., 1988; Roux et al., 1994). Surewicz et al. (1987a) studied the interaction of this myelin protein with phosphatidylglycerol bilayers by IR spectroscopy, and some years later Nabet et al. (1994) applied the same technique to the study of the myelin basic protein-phosphatidic acid system. According to IR data the interaction occurs at many levels of the bilayer structure. The protein appears indeed to interact with the hydrophobic portion of the bilayer, since it induces acyl chain conformational disorder. The phosphate region of the IR spectrum reveals that the hydrogen bond network formed by the charged polar headgroups of phospholipids is replaced, in the presence of the protein, by electrostatic interactions of the protein with the phospholipid headgroups. The myelin basic protein also appears to enhance the second ionization state of the phosphate group at neutral $\mathrm{pH}$. Also, IR spectra of the lipid carbonyl stretching region show evidence that the protein limits the accessibility of water molecules to the interfacial part of the bilayer. As a result of this complex interaction, the myelin basic protein decreases and broadens the gel-fluid transition temperature of phosphatidic acid (but not of phosphatidylglycerol).

Previous studies of the interaction of apocytochrome $c$ with dimyristoylphosphatidylglycerol bilayers (Muga et al., 1991) had shown very similar effects to those of myelin basic protein, a decrease in the frequency of the $\mathrm{CH}_{2}$ stretching bands, suggestive of increased conformational disorder, and a broadening of the gel-fluid phospholipid phase transition.

The case of cardiotoxin interacting with phosphatidic acid bilayers is interesting because, being in principle a small soluble basic protein present in snake venom, it interacts with negativelycharged bilayers in a qualitatively similar way than myelin basic protein or apocytochrome $c$, although with higher potency than those membrane-associated proteins (Désormeaux et al.,
1992). It is nevertheless reasonable that proteins designed to coexist with bilayers in equilibrium have a lower destabilizing capacity than those endowed with toxic properties. Cardiotoxin is also remarkable because, when added to mixed bilayers consisting of phosphatidylcholine and phosphatidic acid, the protein induces lateral phase separation, segregating into phosphatidic acidrich domains. The latter studies were carried out with a phosphatidylcholine containing fully deuterated fatty acyl chains, as in the examples detailed in Section 5.

In the examples described above the lipid was organized in the form of vesicles. However, infrared spectroscopy can also be applied to the study of lipids spread as monolayers at an airwater interface. Subirade et al. (1995) combined IR spectroscopy and fluorescence microscopy in a study of the interaction of a nonspecific wheat lipid transfer protein with phosphatidylglycerol monolayers. This is another example of a basic soluble protein that interacts with phospholipids. The advantage of the monolayer arrangement is that it allows the independent variation of surface pressure as an experimental parameter. In that study, incorporation of wheat lipid transfer protein at low surface pressures (below $20 \mathrm{mN} / \mathrm{m}$ ) leads to an expansion of the phospholipid surface area, together with a decrease in lipid chain order. Neither of these effects are seen at surface pressures above $20 \mathrm{mN} / \mathrm{m}$, although some protein molecules bind the monolayer surface under these conditions, leading to an increased exposure of the lipid ester group to the aqueous environment.

\section{Use of deuterated lipids}

The study of the perturbations introduced by proteins in membrane phospholipids is often influenced by strong protein absorption bands. In this situation, as well as in studies of binary lipid mixtures, the use of perdeuterated lipids provides a mean of circumventing the problem of overlapping signals. Besides, partially deuterated lipids can give information about specific carbon atoms from lipids or can even be used to obtain a quantitative characterization of phospholipid acyl 
chain disordering. The isotopic substitution produces a change in the mass of the molecule that varies the frequency of the absorption bands involved. Thus, the $-\mathrm{CD}_{2}$ stretching bands are shifted from the region $3000-2800 \mathrm{~cm}^{-1}$ to the region 2300-2000 $\mathrm{cm}^{-1}$, free from interfering absorption bands. In perdeuterated acyl chains, the antisymmetric and symmetric bands appear around 2195 and $2090 \mathrm{~cm}^{-1}$, respectively, at variance with the positions obtained for the $-\mathrm{CH}_{2}$ at around 2920 and $2850 \mathrm{~cm}^{-1}$. Perdeuteration also simplifies the region $1400-700 \mathrm{~cm}^{-1}$ where the amide III band of the proteins and the phosphate group stretching vibrations appear, by removing from that region the $-\mathrm{CH}_{2}$ wagging and rockingtwisting progressions.

Deuterated lipids were first used to study lipid mixtures, and were later applied to the study of either pure lipids in the presence of proteins or of lipid mixtures in the presence of proteins. Lipidprotein studies using a single deuterated lipid were conducted on the effects of glycophorin from human erythrocyte membranes, alamethicin or bacteriorhodopsin on DPPC acyl chains (Mendelsohn and Mantsch, 1986). This work allowed the study of the lipid without interference from the protein amino-acid side chain and confirmed previous studies performed with native lipids according to which, below $T_{\mathrm{m}}$, these molecules cause an increase in the proportion of gauche isomers, whereas above $T_{\mathrm{m}}$, and mainly at high lipid/ protein ratios, a reduction in the proportion of gauche conformers with respect to the pure lipid bilayer is seen. Interestingly, bacteriorhodopsin had no effect at any studied concentration on the rate of acyl chain motion above $T_{\mathrm{m}}$. Previously, binary lipid mixtures interacting with proteins were studied separately for each lipid component by using one of the phospholipids in the perdeuterated form. The study of the interaction of glycophorin with a mixture of DMPC- $\mathrm{d}_{54} /$ DPPC (Dluhy et al., 1983) showed that there was no lipid phase separation and that the protein had a more pronounced effect on DPPC than on DMPC. Also, the influence of the phospholipid headgroup on this interaction was tested by using a 30:70 PS/DPPC- $d_{62}$ binary mixture with glycophorin (Mendelsohn et al., 1984a). It was demonstrated that the protein interacted preferentially with PS leading to the appearance of bulk lipid enriched in DPPC- $\mathrm{d}_{62}$. The effect of sarcoplasmic reticulum $\mathrm{Ca}^{2+}$-ATPase on different binary lipid mixtures has also been studied. When the protein is inserted into a mixture of DOPC/ DPPC- $\mathrm{d}_{62}$, a lipid mixture that exhibits gel phase immiscibility, the infrared results are consistent with a preferential selection of DOPC in the vicinity of the protein. However, in lipid mixtures that mix well such as POPE/DPPC- $\mathrm{d}_{62}$ no preferential selection of the lipids towards the protein is seen (Mendelsohn et al., 1984b). The relationship between lipid miscibility and preferential effect of protein in a lipid mixture was later confirmed in a study of the effect of $\mathrm{Ca}^{2+}$-ATPase on SOPC/ DPPC-d ${ }_{62}$ (Jaworsky and Mendelsohn, 1985). The intrinsic protein apocytochrome $c$ affects a mixture of DMPC- $\mathrm{d}_{54} / \mathrm{DMPG}$ in a similar fashion for both lipids (Muga et al., 1991), but the effect upon DMPG is greatly attenuated when compared to the effect of apocythchrome on DMPG alone.

Natural membranes are complex mixtures of lipids and proteins, among other components. Comparison of isolated membrane lipids with the same molecules in their native state may give indications of the in vivo influence of proteins on the lipid properties. However, the complexity of the natural membranes hamper their analysis thus very few studies have been undertaken with these systems. Acholeplasma laidlawii B is a mycoplasma with a thin cell wall and a single membrane system. This microorganism is capable of regulating the fluidity of its plasma membrane when the external fatty acid source is varied. It readily incorporates perdeuterated fatty acids into its membrane lipids, providing a direct probe of the membrane structure via the $\mathrm{C}-\mathrm{D}$ stretching bands. It has been shown that even if the temperature-dependent behaviour shows the same general pattern in the intact and deproteinated membranes, the profiles of the gel-to-liquid-crystal phase transitions are different in the native membrane and the extracted lipids. At temperatures within the range of the phase transition, the live mycoplasma keeps the fluidity of its plasma membrane at a much higher value than that of the isolated membrane (Casal et al., 1980). 
Selectively deuterated lipids can be used to study the properties and dynamics of a specific region of the membrane. The $\mathrm{C}-\mathrm{D}$ rocking $\mathrm{ab}$ sorption of selectively deuterated lipids has been used to quantify the percentage of gauche rotamers (Mendelsohn et al., 1989). In bilayers composed of $6,6,6^{\prime}, 6^{\prime}-\mathrm{d}_{4}$ DPPC/gramicidin A it can be appreciated that the protein induces conformational disorder in the lipid gel phase and orders the crystalline phase. Thus, at $34^{\circ} \mathrm{C}$, below $T_{\mathrm{m}}$, gramicidin $\mathrm{D}$ insertion in a 10:1 lipid:protein ratio causes the total gauche rotamer percentages to increase from $1.7 \%$ in pure lipid vesicles to $9.8 \%$ in the lipid/protein system. In the same mixture above $T_{\mathrm{m}}$, gauche rotamers percentages are $32.3 \%$ for the pure lipid and $15.0 \%$ for the mixture (Davies et al., 1990).

Lipids deuterated in the fatty acyl methyl groups have also been used to characterize the effect of the protein on the lipid moiety of artificial membranes. This label is intended to provide information on lipid dynamics in the region of contact between monolayers. It is of interest to compare the perturbations induced by an intrinsic and by an extrinsic protein and with the effect of cholesterol in this region. Gramicidin A is a hydrophobic peptide that can be readily incorporated into phospholipid bilayers and is often used as a model integral protein. The interaction of gramicidin A with phosphatidylcholines widens the gel-to-fluid transition of the lipid but has little effect on the phospholipid order above $T_{\mathrm{m}}$. Cytochrome $c$ is an example of a peripheral or extrinsic protein although it is known to interact as well with the hydrophobic moieties of phospholipids. Cytochrome $c$ modifies but slightly the amplitude or width of the lipid phase transition as expected from peripheral proteins. The phase transition profiles corresponding to the $\mathrm{CH}_{2}$ and $\mathrm{CD}_{3}$ symmetric vibration of DMPC- $\mathrm{d}_{3}$ in pure lipid, in DMPC$\mathrm{d}_{3} /$ gramicidin $\mathrm{A}$ and in DMPC- $\mathrm{d}_{3} /$ cytochrome $c$ mixtures are shown in Fig. 3. Fig. 3A shows the profiles corresponding to the phase transition of the $\mathrm{CH}_{2}$ vibrational band whereas Fig. 3B shows the $\mathrm{CD}_{3}$ vibrations corresponding to the terminal methyl group of the acyl chains. It can be seen that in the acyl chain the phase transition profile of the pure lipid is broadened by the presence of gramicidin A whereas it is hardly affected by cytochrome $c$. The onset of the transition is not perturbed, but the temperature at which the transition is completed, is affected by the protein and in this case there is no difference between both proteins. In the methyl end the effect is more pronounced for gramicidin, specially below $T_{\mathrm{m}}$ where an increase in protein-induced disorder is clearly seen. Well above $T_{\mathrm{m}}$ the disorder is similar with or without proteins, but whereas the profile for cytochrome $c$ is close to that of the pure lipid, the profile for gramicidin A is different. The differential effect of the intrinsic or extrinsic proteins on the lipid bilayers shown by the deuterated lipids can also be seen by looking at other regions of DMPC as shown in Fig. 4. In the interfacial region, the $\mathrm{C}=\mathrm{O}$ stretching band is not affected by the presence of gramicidin, whereas a shift in band position is observed in the presence of cytochrome $c$ (Fig. 4A). A similar effect is observed in the polar region where the antisymmetric $\mathrm{PO}_{2}^{-}$band position shifts to higher wavenumbers in the presence of cytochrome $c$ indicating some dehydration of the polar heads (Fig. 4B). It can be concluded that vibrational parameters corresponding to fatty acyl methyl end groups in phospholipid bilayers are sensitive to the physical state of the lipids and reflect in different, specific ways, perturbations produced by intrinsic and peripheral proteins when incorporated to the bilayers.

A further application of specifically deuterated lipids in the study of lipid-protein interactions is the in situ insertion of deuterium atoms into the fatty acyl chains of biological membranes. A hydrogenation catalyst in a gaseous $\mathrm{D}_{2}$ atmosphere aids the insertion of deuterium labels into the saturated chains that form upon reduction of $\mathrm{C}=\mathrm{C}$ bonds. This method has been used in native rabbit sarcoplasmic reticulum membranes and in purified phospholipid/ $\mathrm{Ca}^{2+}$-ATPase vesicles (Szalontai et al., 1994). A segregation of particular lipid populations that are excluded from the vicinity of the protein is observed, in agreement with previous work on ATPase reconstituted in binary mixtures of lipids. 

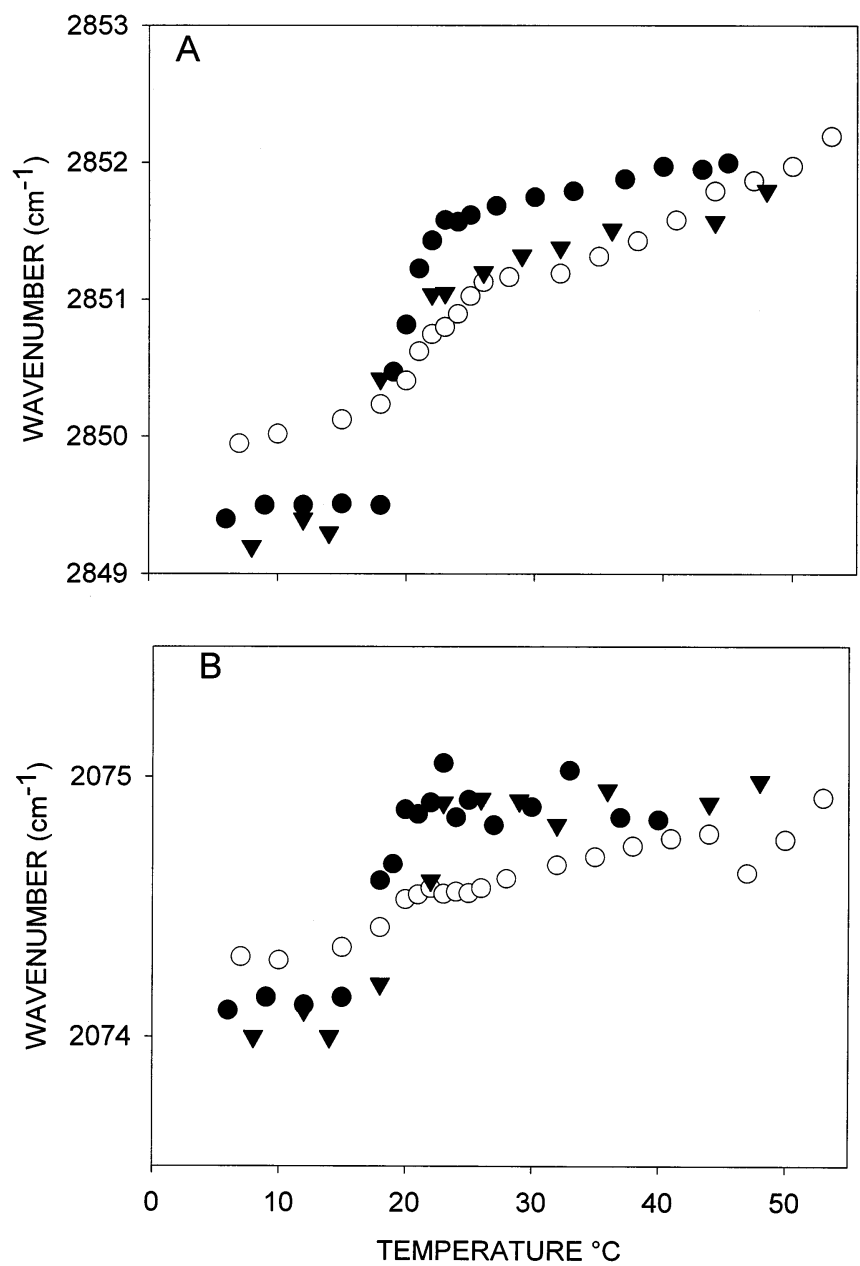

Fig. 3. Thermal profiles corresponding to the position of a $\mathrm{CH}_{2}$ stretching band (A) and $\mathrm{CD}_{3}$ band (B) corresponding to pure DMPC-d $_{3}(\bullet)$, DMPC-d ${ }_{3}:$ gramicidin A 1:1 (O) and DMPC-d d $_{3}:$ cytochrome $c$ 2. 5:1 ( $)$.

\section{The pulmonary lung surfactant}

The pulmonary lung surfactant is a mixture of phospholipids and at least four specific proteins, that lines the air-lung interface of alveoli, where it acts to reduce surface tension, counteracting the tendency of alveoli to collapse at the end of expiration and facilitating gas transport (Bourbon, 1991). Adequate production of surfactant in fetal lung is very important for initiation of breathing at birth. Insufficient production of lung surfactant, as it often occurs in premature infants, may lead to insufficient pulmonary expansion, known as the respiratory distress syndrome (Avery and Mead, 1959). Thus lung surfactant has been extensively studied from several points of view, including IR spectroscopy.

The lipids of lung surfactant, and in particular their perturbation by a synthetic surfactant protein B fragment (SP-B 53-78 diacetamido methyl) have been examined by Krill et al. (1994) using thermal perturbation infrared spectroscopy. A three-component lipid matrix consisting of DPPC, POPG and palmitic acid, that appears to emulate rather closely natural lung surfactant, was used. Lipidic vesicles containing natural and 

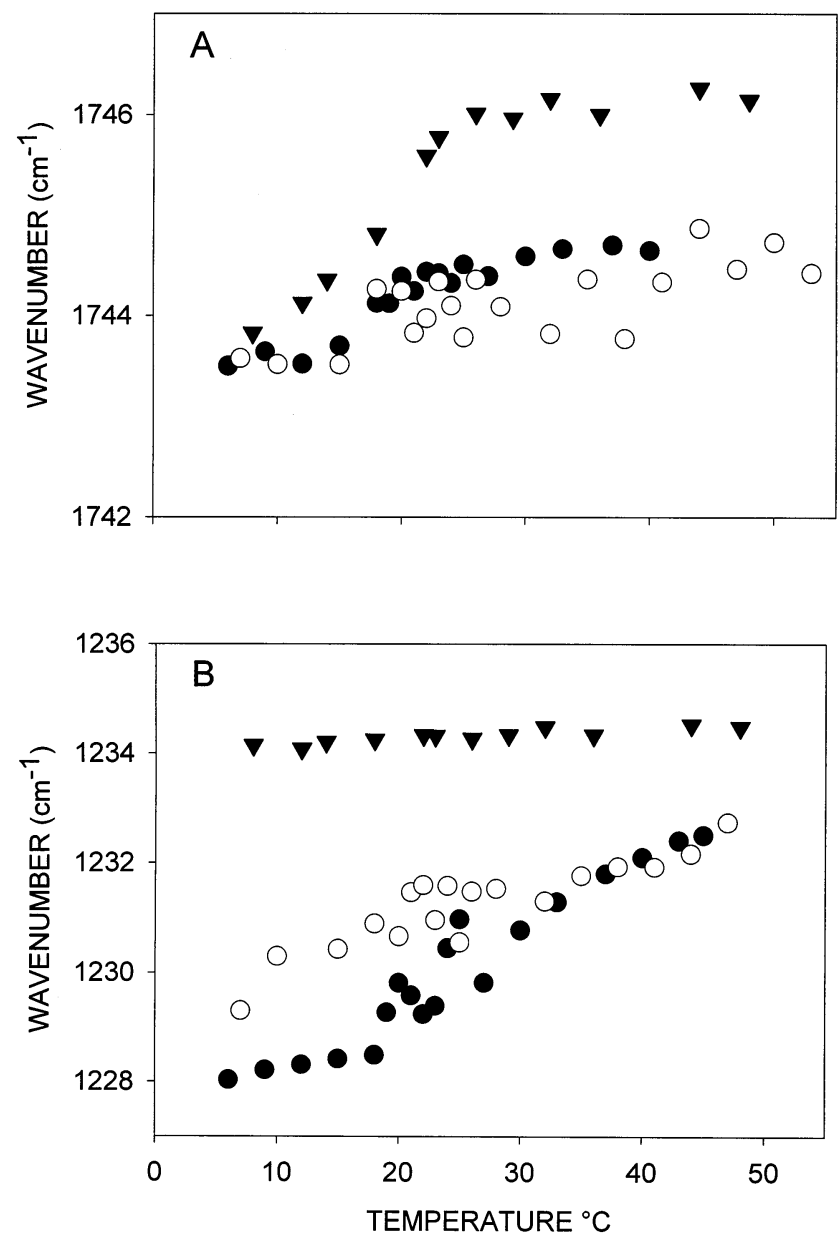

Fig. 4. Thermal profiles of the same samples as in Fig. 3, pure DMPC- $\mathrm{d}_{3}(\bullet)$, DMPC-d $\mathrm{d}_{3}$ :gramicidin A ( $\left.\bigcirc\right)$ and DMPC$\mathrm{d}_{3}$ :cytochrome $c(\boldsymbol{\nabla})$, but in the regions corresponding to the $\mathrm{C}=\mathrm{O}$ stretching band (A) and the $\mathrm{PO}_{2}^{-}$asymmetric stretching band (B).

perdeuterated acyl chains were included in the study. The spectral data reveal that peptide addition results in a significant increase in the acyl chain order of the phospholipids, as measured by the position of the $\mathrm{C}-\mathrm{H}$ stretching bands. An increase in the gel-fluid transition temperature of these phospholipids is also observed, while palmitic acid is hardly affected by the peptide in any aspect. The data would be compatible with a peptide interacting strongly with the phospholipid headgroups, rather than penetrating the hydrophobic region.

Rabbit lung surfactant has also been studied by
IR from a different point of view, recording spectra of bronchoalveolar lavage (Knells et al., 1995). In this study IR is applied to the simultaneous study of proteins and lipids in the samples, and a comparison with biochemical assays demonstrates the capacity of this technique to quantitate lipid/ protein ratios in biological samples. From the point of view of lipid-protein interactions, the authors study the relatively unexplored phosphate stretching bands in the $1000-1300 \mathrm{~cm}^{-1}$ region. After separation of lipid and protein components the asymmetric phosphate stretching band that was observed at $1240 \mathrm{~cm}^{-1}$ in whole lung surfac- 
tant samples was shifted to $1227 \mathrm{~cm}^{-1}$. This further supports the idea that it is the phospholipid headgroups that are interacting with the surfactant proteins.

\section{The human low density lipoprotein}

Human serum low density lipoprotein is a major carrier of serum cholesterol in humans. It is described as a sphaerical particle containing a hydrophobic core of cholesteryl esters and triglycerides surrounded by an amphipathic monolayer of phospholipid and cholesterol in which a single molecule of apolipoprotein B (apo B) is located (Deckelbaum et al., 1977). The core-associated lipids have been shown to undergo an order-disorder transition near human body temperature, the actual transition temperature being determined by the lipid composition (Yang et al., 1986).

Infrared spectroscopy was first applied to the study of apo B by Scanu et al. (Scanu et al., 1969) to examine its thermal behaviour. Later, IR was applied to apo B studies qualitatively using resolution-enhanced techniques (Herzyk et al., 1987) or quantitatively using curve fitting of deconvoluted spectra (Goormaghtigh et al., 1989). Also the structure of the lipid-attached protein has been described by IR in combination with proteolytic digestion (Goormaghtigh et al., 1993). Bañuelos et al. (1995) have investigated conformational changes induced in apo B by changes in temperature and in ionic strength, and taken advantage of the IR ability to record data on lipid and protein moieties simultaneously. This allows the direct study of surface-core relationships in the lipoprotein. Bañuelos et al. have used for this purpose a component of the amide I band with a maximum at $1618 \mathrm{~cm}^{-1}$, corresponding to about $24 \%$ of the total amino acid residues, and assigned to a $\beta$ strand, i.e. an extended chain not forming a $\beta$-sheet with other chains but interacting via a different hydrogen-bonding pattern with other structures. The thermal behaviour of the 1618 $\mathrm{cm}^{-1}$ component is different from that of the other apo B amide I components and, interestingly, it follows a pattern similar to the methylene

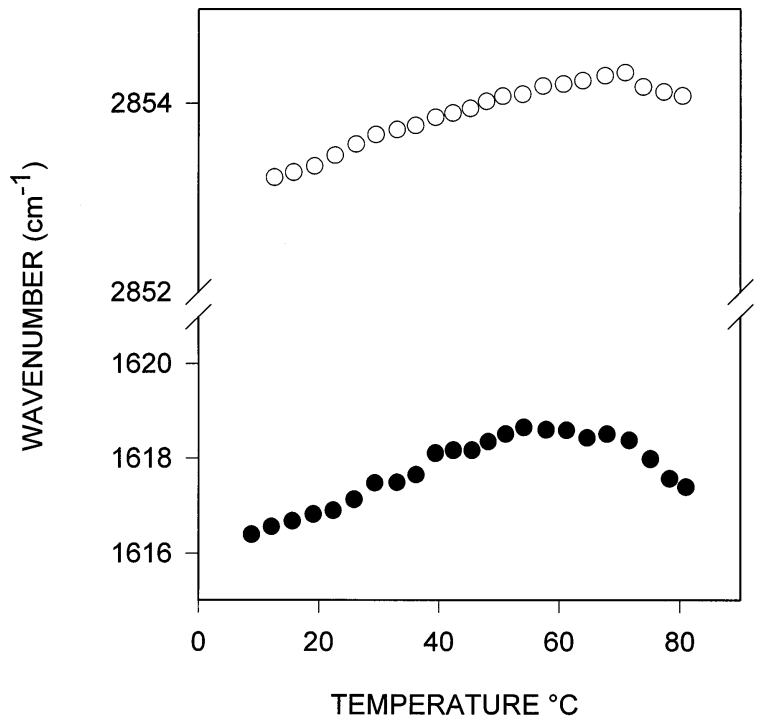

Fig. 5. Thermal profile corresponding to the band located at $1618 \mathrm{~cm}^{-1}(\bullet)$ arising from apolipoprotein $\mathrm{B}$ and the band at around $2850 \mathrm{~cm}^{-1}(\bigcirc)$ due to the $\mathrm{CH}_{2}$ stretching band of the lipid moiety. Both bands correspond to the infrared spectrum of a human low density lipoprotein.

stretching of the lipid moiety (Fig. 5). This is interpreted in terms of a protein structure penetrating the monolayer and establishing hydrophobic interactions deeper than the outer charged shell. The combination of amide I band decomposition and simultaneous perturbation of the lipid and protein moieties provides certainly a novel and powerful method for studying protein-lipid interactions at the molecular level.

\section{Studies with native membranes}

Most of the infrared studies of membrane lipids have been accomplished with model or isolated membranes because of the complexity of cells. The first studies in native membranes were performed on live Acholeplasma laidlawii cells grown on a deuterated fatty acid diet (Cameron et al., 1985). The region $2300-2000 \mathrm{~cm}^{-1}$ contains only absorption bands originating from $\mathrm{CD}_{2}$ stretching vibration. The response of live cells to temperature changes differs drastically from that of the 


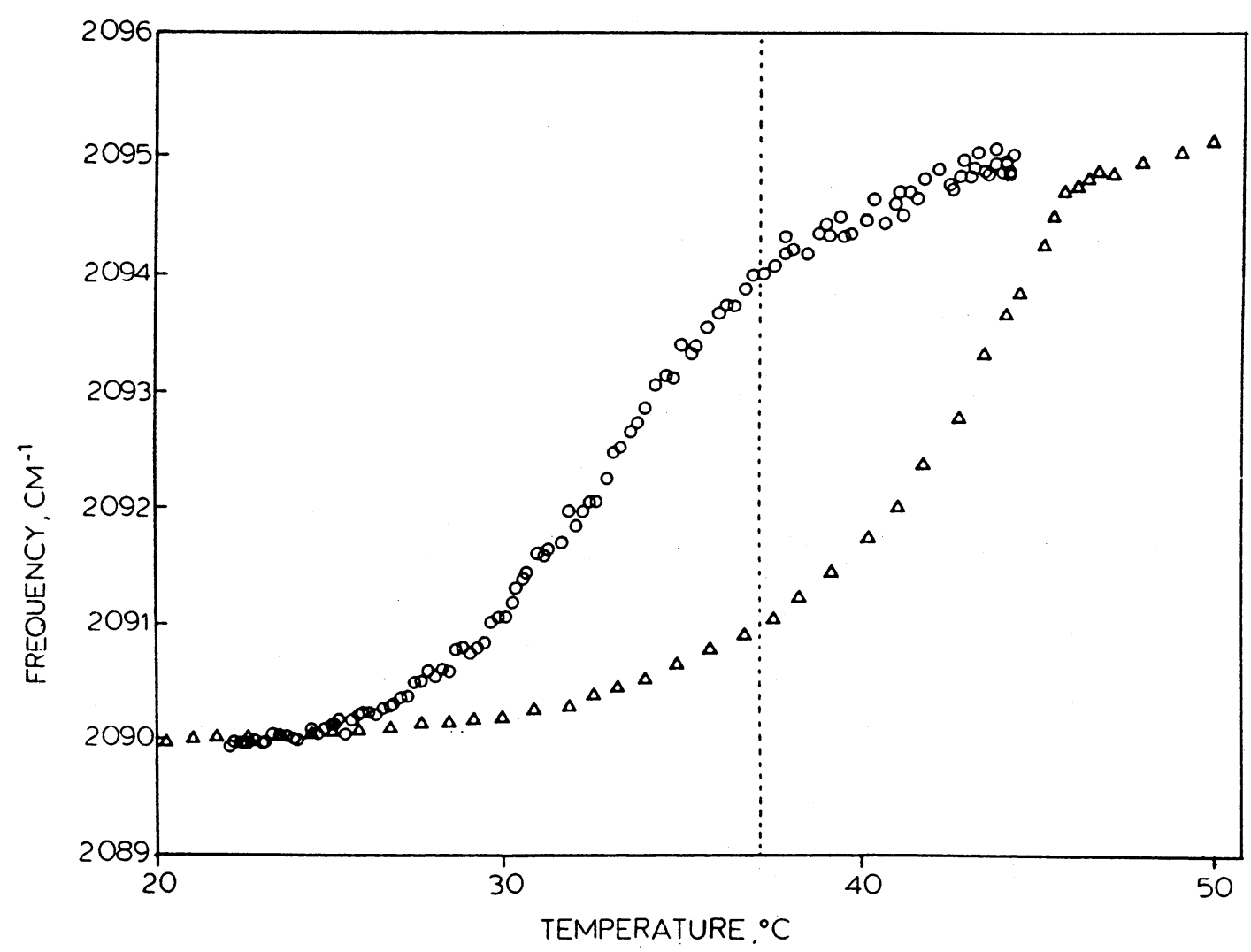

Fig. 6. Comparison between the temperature-dependence of the frequency of the $\mathrm{CD}_{2}$ symmetric stretching band of membranes labeled with perdeuteropalmitoyl lipids in live Acholeplasma laidlawii cells $(\bigcirc)$ and in isolated membranes $(\triangle)$; the temperature of growth, i.e. $37^{\circ} \mathrm{C}$ is marked by a dotted line (Cameron et al., 1985).

isolated membranes. The fractional population of the liquid crystalline phase at any given temperatures is always higher in membranes of live cells and the transition has a higher degree of cooperativity than in the lipids of isolated membranes (Fig. 6). More recently, the conformational order of $A$. laidlawii has been studied looking at the $\mathrm{CH}_{2}$ wagging progression (Moore et al., 1993). Conformational order in the live cells and in the isolated membranes was virtually identical over the range of cell viability $\left(5-40^{\circ} \mathrm{C}\right)$. In contrast, the membrane lipid extracts show much more conformational disorder from 5 to $25^{\circ} \mathrm{C}$ than either the live cells or the membranes. This work yielded substantially different results than the previous one. The differences have been attributed either to differences in experimental conditions or to the use of perdeuterated fatty acids in the previous studies.
The state of order of Gram-negative bacterial membranes in vivo has also been studied by infrared spectroscopy monitoring the frequency shifts of the acyl chain methylene symmetric stretching band (Schultz and Naumann, 1991). Cells grown at different temperatures yielded distinct transition temperature profiles showing the adaptation of the 'state of order' and 'fluidity' of bacterial membranes to varying growth temperatures.

The study of the lipids of isolated native membranes and intact cells may also be carried out by chemically modifying their phospholipid environment using a water soluble hydrogenation catalyst in a deuterium-containing environment (Török et al., 1993) for the in situ insertion of deuterium atoms into the fatty acyl chains of biological membranes. 
Various aspects of the biochemistry of the vertebrate eye have been examined by IR spectroscopy of native membranes. Lamba et al. (1994) studied by this technique the phospholipid composition and structure of disk and plasma membranes purified from bovine rod outer segments. The outer segments of photoreceptor rod cells contain in their membranes the biochemical machinery necessary for the transduction of photons into electrical signals as a part of the visual excitation process. Extending the early studies by Rothschild et al. (1980), Lamba and co-workers observed that both disk and plasma membranes lack sphingophospholipids, in contrast to the lens membranes. Comparison of intact rod outer segment membranes with liposomes formed with their extracted lipids in the $1700-1760 \mathrm{~cm}^{-1}$ region suggests that rhodopsin increases hydrogen bonding between lipid carbonyls and bilayer water molecules. Rhodopsin does also influence the lipid headgroup structure, as revealed by changes in the phosphate stretching bands (1000-1300 $\mathrm{cm}^{-1}$ ). Finally, the broadening and frequency downshift of the $1468 \mathrm{~cm}^{-1}$ band, attributed to $\mathrm{CH}_{2}$ scissoring, in the presence of protein is interpreted by the authors as a result of the interaction of rhodopsin with the lipid acyl chain.

In contrast with these observations, Sato et al. (1996), studying an anatomically related, but functionally different, system, namely human and bovine lens membranes, could not find any difference in the IR spectra of human lens lipids (band centred at $\sim 2850 \mathrm{~cm}^{-1}$ ) in the presence and absence of intrinsic proteins. This observation is against the main body of experimental evidence in the field of lipid-protein interactions, and it should be taken with precaution in view of the difficulties in obtaining suitable amounts of samples, and of their heterogeneity. In the same study, Sato et al. find that the extrinsic protein $\alpha$-crystallin does not modify the $\mathrm{CH}_{2}$ symmetric stretching band frequency of extracted bovine lens lipids when the protein in solution is incubated with sonicated lipid dispersions.

In summary, the observations in native membranes are in general agreement with the conclusions reached in model systems, and support the validity of IR spectroscopy as a tool for the simultaneous study of lipid and protein components in membraneous systems.

\section{Molecular recognition}

Specific and reversible protein docking at lipid interfaces has been demonstrated by external reflection FT-IR spectroscopy at the air-water interface (Schmitt et al., 1996). For this purpose, a histidine-tagged fusion protein (a heat shock tomato protein with a $\mathrm{C}$-terminal end containing six His residues) was used, together with a novel class of synthetic lipids containing a nitrilotriacetic acid (a nickel chelator) in the polar headgroups. The lipids (either free or Ni-complexed) were spread onto an air-water interface, while the histidine-tagged protein was injected into the subphase. Nickel unloaded monolayers provide a control for unspecific protein binding. Specific protein docking is marked by the appearance of an IR signal at $3014 \mathrm{~cm}^{-1}$, that the authors attribute to intramolecular hydrogen bonds of the $\mathrm{NH}$ groups, or to an overtone of the amide II absorption. The positions of the lipid $\mathrm{C}-\mathrm{H}$ stretching modes were not affected by the binding of the protein. Only specifically bound heat shock protein retained its biological ability to bind DNA. The protein could be dissociated from the lipid monolayer by various methods, and the dissociation followed by IR spectroscopy. The large number of available histidine-tagged fusion proteins makes the procedure of general interest for the study of molecular mechanisms of recognition at the membrane level.

\section{Concluding remarks}

The available body of experimental evidence allows a number of generalisations to be made on the perturbations caused by proteins on their lipid environment, as seen by infrared spectroscopy:

1. Proteins interacting mainly with the hydrophobic matrix of the lipid bilayer (e.g. integral transmembrane proteins) broaden the gel-toliquid crystal (fluid) phase transition of satu- 
rated bilayers, without modifying the $T_{\mathrm{m}}$ transition temperature. In the gel phase they cause an increase in the proportion of gauche isomers (increased chain disorder). In the fluid state, and particularly at high lipid/protein ratios, there is a slight increase in acyl chain order. Both in the gel and liquid-crystal phases the $\mathrm{C}-\mathrm{H}$ stretching bandwidth is increased, suggesting increased rates of librational chain motion in the presence of the protein. Phosphate stretching bands are not modified by this kind of proteins, at least in zwitterionic phospholipids.

2. Proteins that interact mainly with the polar moiety of phospholipids (e.g. peripheral membrane proteins) tend to bind negatively-charged phospholipids, modifying the network of hydrogen bonds at the bilayer surface, so that the phospholipid polar headgroups exchange hydrogen bonds with the protein, rather than with water. As a result, the bilayer surface is partially dehydrated. The ionization state of the phosphate groups is also modified by the protein, i.e. phosphate protonation is decreased. The $T_{\mathrm{m}}$ gel-to-fluid transition temperature may be shifted. In some cases, protein docking to the lipid-water interface in mixed bilayers may lead to lateral phase separation.

3. In most cases proteins show an intermediate behaviour between the purely hydrophobic and the purely polar interaction, following in different degrees the patterns indicated in the two previous paragraphs.

4. Lipid-protein interactions in native, in situ or isolated, cell membranes do not reveal, when examined by IR spectroscopy, any substantial differences from what is observed in reconstituted model systems.

5. The use of deuterated (and, perhaps, of other isotopically substituted molecules) is particularly useful in IR studies of lipid-protein interaction.

6. Infrared spectroscopy constitutes a unique tool in the study of membrane structure and dynamics, particularly because of its capacity to detect simultaneously signals arising from all the membrane components: proteins, lipids, sugars, and any other.

\section{Acknowledgements}

This work was supported in part by grants from DGICYT (PB 96/01 71), and the University of the Basque Country (UPV042.310-EB219/95).

\section{References}

Anderle, GL., Mendelsohn, R., 1986. Fourier-transform infrared studies of Ca-ATPase/phospholipid interaction: survey of lipid classes. Biochemistry 25, 2174-2179.

Arrondo, J.L.R., Goñi, F.M., 1993. Infrared spectroscopic studies of lipid-protein interactions in membranes. In: Watts, A. (Ed.), Protein-Lipid Interactions. Elsevier Science, Amsterdam, pp. 321-349.

Arrondo, J.L.R., Goñi, F.M., Macarulla, J.M., 1984. Infrared spectroscopy of phosphatidylcholines in aqueous suspension. A study of the phosphate group vibrations. Biochim. Biophys. Acta 794, 165-168.

Avery, M.E., Mead, J., 1959. Am. J. Dis. Child 97, 517-527.

Bañuelos, S., Arrondo, J.L.R., Goñi, F.M., Pifat, G., 1995. Surface-core relationships in human low density lipoprotein as studied by infrared spectroscopy. J. Biol. Chem. 270, 9192-9196.

Bellamy, L.J., 1980. The Infrared Spectra of Complex Molecules. Chapman and Hall, London.

Blume, A., Hübner, W., Messner, G., 1988. Fourier transform infrared spectroscopy of ${ }^{13} \mathrm{C}=\mathrm{O}$-labeled phospholipids. Hydrogen bonding to carbonyl groups. Biochemistry 27, 8239-8249.

Boggs, J.M., Angaraj, G., Koshy, M., 1988. Photolabeling of myelin basic protein in lipid vesicles with the hydrophobic reagent 3-(trifluoromethyl)-3-(m-(125-I)iodophenyl)diazinine. Biochim. Biophys. Acta 937, 1-9.

Bourbon, J.R., 1991. In: Bourbon, J.R. (Ed.), Pulmonary Surfactant: Biochemical, Functional, Regulatory and Clinical Concepts. CRC, Boca Raton, pp. 1-14.

Cameron, D.G., Martin, A., Moffat, D.J., Mantsch, H.H., 1985. Infrared spectroscopic study of the gel-to-liquid-crystal phase transition in live Acholeplasma laidlawii cells. Biochemistry 24, 4355-4359.

Casal, H.L., Mantsch, H.H., 1984. Polymorphic phase behaviour of phospholipid membranes studied by infrared spectroscopy. Biochim. Biophys. Acta 779, 381-401.

Casal, H.L., Cameron, D.G., Smith, I.C.P., Mantsch, H.H., 1980. Acholeplasma laidlawii membranes: a Fourier transform infrared study of the influence of protein on lipid organization and dynamics. Biochemistry 19, 444-451.

Castresana, J., Valpuesta, J.M., Arrondo, J.L.R., Goñi, F.M., 1991. An infrared spectroscopic study of specifically deuterated fatty-acyl methyl groups in phosphatidylcholine liposomes. Biochim. Biophys. Acta 1065, 29-34.

Castresana, J., Fernandez-Ballester, G., Fernandez, A.M., Laynez, J.L., Arrondo, J.L.R., Ferragut, J.A., GonzalezRos, J.M., 1992. Protein structural effects of agonist bin- 
ding to the nicotinic acetylcholine receptor. FEBS Lett. $314,171-175$.

Chapman, D., Gómez-Fernández, J.C., Goñi, F.M., 1979. Intrinsic protein-lipid interactions: physical and biochemical evidence. FEBS Lett. 98, 211-223.

Chapman, D., Gómez-Fernández, J.C., Goñi, F.M., 1982. The interaction of intrinsic proteins and lipids in biomembranes. Trends Biochem. Sci. 7, 67-70.

Cortijo, M., Alonso, A., Gómez-Fernández, J.C., Chapman, D., 1982. Intrinsic protein-lipid interactions. Infrared spectroscopic studies of gramicidin A, bacteriorhodopsin and $\mathrm{Ca}^{2+}$-ATPase in biomembranes and reconstituted systems. J. Mol. Biol. 157, 597-618.

Davies, M.A., Brauner, J.W., Schuster, H.F., Mendelsohn, R., 1990. A quantitative infrared determination of acyl chain conformation in gramicidin/dipalmitoylphosphatidylcoline mixtures. Biochem. Biophys. Res. Commun. 168, 85-90.

Deckelbaum, R.J., Shipley, G.G., Small, D.M., 1977. Structure and interactions of lipids in human plasma low density lipoproteins. J. Biol. Chem. 252, 744-754.

Désormeaux, A., Laroche, G., Bougis, P.E., Pézolet, M., 1992. Characterization by infrared spectroscopy of the interaction of a cardiotoxin with phosphatidic acid and with binary mixtures of phosphatidic acid and phosphatidylcholine. Biochemistry 31, 12173-12182.

Dluhy, R.A., Mendelsohn, R., Casal, H.L., Mantsch, H.H., 1983. Interaction of dipalmitoylphosphatidylcholine and dimyristoylphosphatidylcholine- $\mathrm{d}_{54}$ mixtures with glycophorin. A Fourier transform infrared investigation. Biochemistry 22, 1170-1177.

Fernandez-Ballester, G., Castresana, J., Fernandez, A.M., Arrondo, J.L.R., Ferragut, J.A., Gonzalez-Ros, J.M., 1994. A role for cholesterol as a structural effector of the nicotinic acetylcholine receptor. Biochemistry 33, 4065-4071.

Flach, C.R., Mendelsohn, R., 1993. A new infrared spectroscopic marker for cochleate phases in phosphatidylserinecontaining model membranes. Biophys. J. 64, 1113-1121.

Fringeli, U.P., Günthard, H.H., 1981. Infrared membrane spectroscopy. In: Grell, E. (Ed.), Membrane Spectroscopy. Springer, Berlin, pp. 270-332.

Goormaghtigh, E., DeMeutter, J., Vanloo, B., Brasseur, R., Rosseneu, M., Ruysschaert, J.M., 1989. Evaluation of the secondary structure of apo B-100 in low density lipoprotein (LDL) by infrared spectroscopy. Biochim. Biophys. Acta 1006, 147-150.

Goormaghtigh, E., Cabiaux, V., DeMeutter, J., Rosseneu, M., Ruysschaert, J.M., 1993. Secondary structure of the particle associating domain of apolipoprotein B-100 in low density lipoprotein by attenuated total reflection infrared spectroscopy. Biochemistry 32, 6104-6110.

Herzyk, E., Lee, D.C., Dunn, R.C., Bruckdorfer, K.R., Chapman, D., 1987. Changes in the secondary structure of apolipoprotein B-100 after $\mathrm{Cu}^{2+}$-catalysed oxidation of human low density lipoproteins monitored by Fourier transform infrared spectroscopy. Biochim. Biophys. Acta 922, 145-154.

Jaworsky, M., Mendelsohn, R., 1985. Fourier transform in- frared studies of CaATPase partitioning in phospholipid mixtures of 1,2-dipalmitoylphosphatidycholine- $\mathrm{d}_{62}$ with 1-palmitoyl-2-oleyl-phosphatidylethanolamine and 1-stearoyl - 2 - oleylphosphatidylcholine. Biochemistry 24, 34223428.

Knells, G., Ahmed, M.K., Das, R.M., Oulton, M.R., Mantsch, H.H., Scott, J.E., 1995. Fourier-transform infrared spectroscopic analysis of rabbit lung surfactant: subfraction-associated phospholipid and protein profiles. Chem. Phys. Lipids 77, 193-201.

Krill, S.L., Gupta, S.L., Smith, T., 1994. Pulmonary lung surfactant synthetic peptide concentration-dependent modulation of DPPC and POPG acyl chain order in a DPPC:POPG:palmitic acid lipid mixture. Chem. Phys. Lipids 71, 47-59.

Lamba, O.P., Borchman, D., Garner, W.H., 1994. Spectral characterization of lipid peroxidation in rabbit lens membranes induced by hydrogen peroxide in the presence of $\mathrm{Fe}^{2+} / \mathrm{Fe}^{3+}$ cations: a site-specific catalyzed oxidation. Free Radic. Biol. Med. 16, 591-601.

Lee, D.C., Chapman, D., 1986. Infrared spectroscopic studies of biomembranes and model membranes. Biosci. Rep. 6, $235-256$.

Levin, I.W., Mushayakarara, E., Bittman, R., 1982. Vibrational assignment of the $s n 1$ and $s n 2$ carbonyl stretching modes of membrane phospholipids. J. Raman Spectroscop. 13, 231-234.

Lewis, R.N.A.H., McElhaney, R.N., 1992. Structures of the subgel phases of $n$-saturated diacyl phosphatidylcholine bilayers: FTIR spectroscopic studies of ${ }^{13} \mathrm{C}=\mathrm{O}$ and ${ }^{2} \mathrm{H}$ labeled lipids. Biophys. J. 61, 63-77.

Lewis, R.N.A.H., McElhaney, R.N., Pohle, W., Mantsch, H.H., 1994. Components of the carbonyl stretching band in the infrared spectra of hydrated 1,2-diacylglycerolipid bilayers. A re-evaluation. Biophys. J. 67, 2367-2375.

Mantsch, H.H., McElhaney, R.N., 1991. Phospholipid phase transitions in model and biological membranes as studied by infrared spectroscopy. Chem. Phys. Lipids 57, 213-226.

Mendelsohn, R., Mantsch, H.H., 1986. Fourier transform infrared studies of lipid-protein interaction. In: Watts, A., DePont, J.J.H.H.M. (Eds.), Progress in Protein-Lipid Interactions, vol. 2. Elsevier, Amsterdam, pp. 103-146.

Mendelsohn, R., Dluhy, R.A., Taraschi, T., Cameron, D.G., Mantsch, H.H., 1981. Raman and Fourier transform infrared spectroscopic studies of the interaction between glycophorin and dimyristoylphosphatidylcholine. Biochemistry 20, 6699-6706.

Mendelsohn, R., Brauner, J.W., Faines, L., Mantsch, H.H., Dluhy, R.A., 1984a. Calorimetric and Fourier transform infrared spectroscopic studies on the interaction of glycophorin with phosphatidylserine/dipalmitoylphosphatidylcholine- $\mathrm{d}_{62}$ mixtures. Biochim. Biophys. Acta 774, 237-246.

Mendelsohn, R., Anderle, G., Jaworsky, M., Mantsch, H.H., Dluhy, R.A., 1984b. Fourier transform infrared spec- 
troscopy studies of lipid-protein interaction in native and reconstituted sarcoplasmic reticulum. Biochim. Biophys. Acta 775, 215-224.

Mendelsohn, R., Davies, M.A., Brauner, J.W., Schuster, H.F., Dluhy, R.A., 1989. Quantitative determination of conformational disorder in the acyl chains of phospholipid bilayers by infrared spectroscopy. Biochemistry 28, 8934-8939.

Moore, D.J., Wyrwa, M., Reboulleau, C.P., Mendelsohn, R., 1993. Quantitative IR studies of acyl chain conformational order in fatty acid homogeneous membranes of live cells of Acholeplasma laidlawii B. Biochemistry 32, 62816287.

Muga, A., Mantsch, H.H., Surewicz, W.K., 1991. Apocytochrome $c$ interaction with phospholipid membranes studied by Fourier-transform infrared spectroscopy. Biochemistry 30, 2629-2635.

Nabet, A., Boggs, J.M., Pezolet, M., 1994. Study by infrared spectroscopy of the interaction of bovine myelin basic protein with phosphatidic acid. Biochemistry 33, 1479214799.

Rothschild, K.J., Clark, N.A., Rosen, K.M., Sanches, R., Hsiao, T.L., 1980. Spectroscopic study of photoreceptor membrane incorporated into a multilamellar film. Biochem. Biophys. Res. Commun. 92, 1266-1272.

Roux, M., Nezil, F.A., Monck, M., Bloom, M., 1994. Fragmentation of phospholipid bilayers by myelin basic protein. Biochemistry 33, 307-311.

Sunder, S., Cameron, D.G., Casal, H.L., Boulanger, Y., Mantsch, H.H., 1981. Infrared and Raman spectra of specifically deuterated 1,2-dipalmitoyl-sn-glycero-3-phosphocholines. Chem. Phys. Lipids 28, 137-148.

Sato, H., Borchman, D., Ozaki, Y., Lamba, O.P., Byrdwell, W.C., Yappert, M.C., Paterson, C.A., 1996. Lipidprotein interactions in human and bovine lens membranes by Fourier transform Raman and infrared spectroscopies. Exp. Eye Res. 62, 47-53.

Scanu, A., Pollard, H., Hirz, R., Kothary, K., 1969. On the conformational instability of human serum low density lipoproteins: effect of temperature. Proc. Natl. Acad. Sci. USA $62,171-178$.

Schmitt, L., Bohanon, T.M., Denzinger, S., Ringsdorf, H., Tampé, R., 1996. Specific protein docking to chelator lipid monolayers monitored by FT-IR spectroscopy at the air-water interface. Angew. Chem. Engl. 35, 317-320.

Schultz, C., Naumann, D., 1991. In vivo study of the state of order of the membranes of Gram-negative bacteria by Fourier-transform infrared spectroscopy (FT-IR). FEBS Lett. 294, 43-46.

Subirade, M., Salesse, C., Marion, D., Pézolet, M., 1995. Interaction of a nonspecific wheat lipid transfer protein with phospholipid monolayers imaged by fluorescence microscopy and studied by infrared spectroscopy. Biophys. J. 69, 974-988.

Surewicz, W.K., Moscarello, M.A., Mantsch, H.H., 1987a. Fourier transform infrared spectroscopic investigation of the interaction between myelin basic protein and dimyristoylphosphatidylglycerol bilayers. Biochemistry 26, 38813886.

Surewicz, W.K., Epand, R.M., Orlowski, R.C., Mantsch, H.H., 1987b. Structural properties of acidic phospholipids in complexes with calcitonin: a Fourier transform infrared spectroscopic investigation. Biochim. Biophys. Acta 899, 307-310.

Szalontai, B., Vigh, L., Joó, F., Senak, L., Mendelsohn, R., 1994. In situ modification of the phospholipid environment of native rabbit sarcoplasmic reticulum membranes. Biochem. Biophys. Res. Commun. 200, 246-252.

Tanford, A., 1980. Formation of Micelles and Biological Membranes. The Hydrophobic Effect. Wiley, New York, pp. 159-164.

Török, Z., Szalontai, B., Joó, F., Wistrom, C.A., Vigh, L., 1993. Homogeneous catalytic deuteration of fatty acyl chains as a tool to detect lipid phase transitions in specific membrane domains: a Fourier transform infrared spectroscopic study. Biochem. Biophys. Res. Commun. 192, 518-524.

Watts, A., (Ed.), 1993. Protein-Lipid Interactions. Elsevier Science, New York.

Watts, A., DuPont, J.J.H.H.M. (Eds.), 1986. Progress in Protein-Lipid Interactions. Elsevier, Amsterdam.

Yang, C.Y., Chen, S.H., Gianturco, S.H., et al., 1986. Sequence, structure, receptor-binding domains and internal repeats of human apolipoprotein B-100. Nature 323, 738742 . 